\title{
The Accurate Location Estimation of Sensor Node Using Received Signal Strength Measurements in Large-Scale Farmland
}

\author{
Yisheng Miao, ${ }^{1,2,3}$ Huarui $W u \mathbb{D}^{1,2,3}$ and Lihong Zhang ${ }^{1,2,3}$ \\ ${ }^{1}$ National Engineering Research Center for Information Technology in Agriculture, Beijing 100097, China \\ ${ }^{2}$ Beijing Research Center for Information Technology in Agriculture, Beijing Academy of Agriculture and Forestry Sciences, \\ Beijing 100097, China \\ ${ }^{3}$ Key Laboratory for Information Technologies in Agriculture, Ministry of Agriculture, Beijing 100097, China
}

Correspondence should be addressed to Huarui Wu; wuhrnercita@163.com

Received 2 September 2017; Revised 10 January 2018; Accepted 21 January 2018; Published 26 March 2018

Academic Editor: Carlos Ruiz

Copyright ( 2018 Yisheng Miao et al. This is an open access article distributed under the Creative Commons Attribution License, which permits unrestricted use, distribution, and reproduction in any medium, provided the original work is properly cited.

\begin{abstract}
The range measurement is the premise for location, and the precise range measurement is the assurance of accurate location. Hence, it is essential to know the accurate internode distance. It is noted that the path loss model plays an important role in improving the quality and reliability of ranging accuracy. Therefore, it is necessary to investigate the path loss model in actual propagation environment. Through the analysis of experiments performed at the wheat field, we find that the best fitted parametric exponential decay model (OFPEDM) can achieve a higher distance estimation accuracy and adaptability to environment variations in comparison to the traditional path loss models. Based on the proposed OFPEDM, we perform the RSSI-based location experiments in wheat field. Through simulating the location characteristics in MATLAB, we find that for all the unknown nodes, the location errors range from $0.0004 \mathrm{~m}$ to $5.1739 \mathrm{~m}$. The location error in this RSSI-based location algorithm is acceptable in the wide areas such as wheat field. The findings in this research may provide reference for location estimation in large-scale farmland.
\end{abstract}

\section{Introduction}

In recent years, wireless sensor network (WSN) has become a hot research topic. The WSN has application potentials in a variety of fields, such as habitat monitoring, environment monitoring, and target tracking [1]. It is known that the location information of sensor node is a premise for perceiving and collecting data. Hence, location technique plays a crucial role in WSN applications [2]. Due to the constraints of hardware cost and energy consumption, only a few sensor nodes, that is, anchor nodes are configured with location information. Other sensor nodes locate themselves by the internode distance measurements [3-5].

Generally, the WSN location techniques can be classified into two categories, that is, range-free and range-based localization. Concretely speaking, range-free localization can estimate the relative distance by considering the internode connectivity and the network topology relationship. Compared with range-free localization, the range-based localization owns higher accuracy. There are some common range-based approaches, such as time of arrival (TOA) $[6,7]$, time difference of arrival (TDOA) [8], angle of arrival (AOA) $[9,10]$, and received signal strength indication (RSSI) $[11,12]$. Among them, TOA, TDOA, and AOA methods have high accuracy, but they require complex hardware and additional energy consumption [13]. The RSSI-based method utilizes the information provided by radio frequency (RF) apparatus, and does not need additional cost [14]. Hence, WSN systems are apt to adopt the RSSI-based methods to estimate the location of sensor nodes.

Obviously, range measurement is the premise for location, and the precise range measurement is the assurance of accurate location. Hence, it is essential to know the accurate internode distance. The RSSI-based ranging methods can be conveniently implemented since most RF IC chips of WSN nodes can directly provide the information of RSSI [15]. The essence of the RSSI-based ranging is that the average power of radio signal decays with the distance 
TABLE 1: The location error of the unknown nodes.

\begin{tabular}{|c|c|c|c|}
\hline $\begin{array}{l}\text { Unknown } \\
\text { node }\end{array}$ & $\begin{array}{l}\text { The actual } \\
\text { coordinate }\end{array}$ & $\begin{array}{l}\text { The estimated } \\
\text { coordinate }\end{array}$ & $d_{e}$ \\
\hline 1 & $(53.86,99.17)$ & $(53.8547,99.5506)$ & 0.3806 \\
\hline 2 & $(69.16,97.90)$ & $(69.5852,99.1215)$ & 1.2934 \\
\hline 3 & $(75.52,98.05)$ & $(75.4487,98.0031)$ & 0.0853 \\
\hline 4 & $(99.95,98.10)$ & $(100.000,99.5281)$ & 2.0170 \\
\hline 5 & $(44.09,95.62)$ & $(43.9773,95.3976)$ & 0.2493 \\
\hline 6 & $(55.26,95.75)$ & $(55.7546,96.7408)$ & 1.1074 \\
\hline 7 & $(85.72,96.36)$ & $(84.7551,96.7051)$ & 1.0247 \\
\hline 8 & $(98.83,92.95)$ & $(100.000,94.7182)$ & 5.1739 \\
\hline 9 & $(68.53,90.95)$ & $(68.5695,91.7274)$ & 0.7784 \\
\hline 10 & $(61.09,90.00)$ & $(61.4222,90.6762)$ & 0.7534 \\
\hline 11 & $(3.487,88.54)$ & $(3.4802,88.5690)$ & 0.0297 \\
\hline 12 & $(43.33,88.42)$ & $(43.3730,88.5003)$ & 0.0911 \\
\hline 13 & $(58.27,85.49)$ & $(58.4510,85.3064)$ & 0.2573 \\
\hline 14 & $(60.20,85.72)$ & $(60.2110,85.9911)$ & 0.2714 \\
\hline 15 & $(24.11,84.14)$ & $(23.9612,83.8992)$ & 0.2831 \\
\hline 16 & $(15.57,81.90)$ & $(15.5258,81.9665)$ & 0.0798 \\
\hline 17 & $(33.81,83.23)$ & $(33.8486,83.3161)$ & 0.0944 \\
\hline 18 & $(82.41,82.80)$ & $(82.9457,82.9632)$ & 0.5600 \\
\hline 19 & $(93.22,83.51)$ & $(93.7406,83.6743)$ & 0.5459 \\
\hline 20 & $(32.79,80.30)$ & $(32.8270,80.3812)$ & 0.0893 \\
\hline 21 & $(62.28,79.66)$ & $(62.3054,78.8524)$ & 0.8080 \\
\hline 22 & $(83.18,81.03)$ & $(84.0795,81.2976)$ & 0.9385 \\
\hline 23 & $(35.41,78.04)$ & $(35.4324,78.0882)$ & 0.0531 \\
\hline 24 & $(0.8648,72.71)$ & $(0.49024,72.5603)$ & 0.4034 \\
\hline 25 & $(5.144,75.69)$ & $(5.5999,74.4384)$ & 1.3320 \\
\hline 26 & $(9.595,74.75)$ & $(9.7128,75.2122)$ & 0.4770 \\
\hline 27 & $(19.34,75.44)$ & $(19.2682,75.5018)$ & 0.0947 \\
\hline 28 & $(41.44,73.14)$ & $(41.7375 .73 .7226)$ & 0.6542 \\
\hline 29 & $(62.49,73.86)$ & $(62.3447,73.8030)$ & 0.1561 \\
\hline 30 & $(75.51,74.24)$ & $(75.3777,74.3627)$ & 0.1804 \\
\hline 31 & $(74.04,69.28)$ & $(73.7161,69.1931)$ & 0.3354 \\
\hline 32 & $(46.12,63.93)$ & $(46.7284,63.3070)$ & 0.8708 \\
\hline 33 & $(2.363,60.74)$ & $(3.8928,59.2709)$ & 2.1209 \\
\hline 34 & $(9.11,59.40)$ & $(7.4142,61.8907)$ & 3.0131 \\
\hline 35 & $(14.39,60.60)$ & $(14.1920,60.9937)$ & 0.4407 \\
\hline 36 & $(38.62,60.98)$ & $(39.7007,62.2139)$ & 1.6402 \\
\hline 37 & $(45.73,61.81)$ & $(46.6605,61.5310)$ & 0.9714 \\
\hline 38 & $(63.33,62.40)$ & $(63.3864,63.3427)$ & 0.9444 \\
\hline 39 & $(71.56,57.77)$ & $(71.6181,57.6157)$ & 0.1649 \\
\hline 40 & $(89.54,58.25)$ & $(90.0705,58.4220)$ & 0.5577 \\
\hline 41 & $(22.62,53.68)$ & $(22.6618,53.6019)$ & 0.0886 \\
\hline 42 & $(23.48,52.86)$ & $(23.5775,52.6722)$ & 0.2116 \\
\hline 43 & $(74.85,54.33)$ & $(75.0351,53.3995)$ & 0.9488 \\
\hline 44 & $(88.41,54.81)$ & $(88.3739,54.8210)$ & 0.0378 \\
\hline 45 & $(12.40,47.08)$ & $(13.2152,45.4160)$ & 1.8529 \\
\hline 46 & $(95.08,49.76)$ & $(95.1055,49.7480)$ & 0.0281 \\
\hline 47 & $(43.67,43.66)$ & $(43.6975,43.6567)$ & 0.0277 \\
\hline 48 & $(11.08,40.75)$ & $(11.4904,41.2254)$ & 0.6280 \\
\hline
\end{tabular}

TABle 1: Continued.

\begin{tabular}{|c|c|c|c|}
\hline $\begin{array}{l}\text { Unknown } \\
\text { node }\end{array}$ & $\begin{array}{l}\text { The actual } \\
\text { coordinate }\end{array}$ & $\begin{array}{l}\text { The estimated } \\
\text { coordinate }\end{array}$ & $d_{e}$ \\
\hline 49 & $(34.64,41.86)$ & $(34.8080,41.9797)$ & 0.2063 \\
\hline 50 & $(40.18,40.64)$ & $(40.3090,40.7335)$ & 0.1593 \\
\hline 51 & $(45.64,38.46)$ & $(45.6678,38.4831)$ & 0.0362 \\
\hline 52 & $(9.463,32.32)$ & $(9.4613,32.3286)$ & 0.0088 \\
\hline 53 & $(21.93,32.58)$ & $(21.9005,31.8288)$ & 0.7518 \\
\hline 54 & $(25.45,32.42)$ & $(25.3998,32.2357)$ & 0.1910 \\
\hline 55 & $(29.34,30.94)$ & $(29.4190,30.7071)$ & 0.2459 \\
\hline 56 & $(52.30,32.53)$ & $(52.2985,32.5291)$ & 0.0018 \\
\hline 57 & $(54.64,34.67)$ & $(54.6398,34.6697)$ & 0.0004 \\
\hline 58 & $(76.21,34.76)$ & $(76.1933,34.9197)$ & 0.1606 \\
\hline 59 & $(78.14,36.73)$ & $(78.1743,36.4429)$ & 0.2905 \\
\hline 60 & $(89.28,35.65)$ & $(88.9725,36.3353)$ & 0.7511 \\
\hline 61 & $(55.70,26.30)$ & $(55.6268,26.3550)$ & 0.0915 \\
\hline 62 & $(12.70,23.22)$ & $(12.7013,23.2090)$ & 0.0110 \\
\hline 63 & $(16.69,18.81)$ & $(16.6905,18.8049)$ & 0.0051 \\
\hline 64 & $(36.90,20.83)$ & $(37.0410,21.0094)$ & 0.2282 \\
\hline 65 & $(39.31,17.90)$ & $(39.2496,17.3705)$ & 0.5329 \\
\hline 66 & $(48.89,22.03)$ & $(49.1628,21.9024)$ & 0.3012 \\
\hline 67 & $(54.09,20.77)$ & $(54.1833,20.7222)$ & 0.1048 \\
\hline 68 & $(68.06,23.37)$ & $(68.1223,23.4265)$ & 0.0841 \\
\hline 69 & $(76.96,23.41)$ & $(76.9574,22.7212)$ & 0.6889 \\
\hline 70 & $(28.33,13.38)$ & $(28.3747,13.5905)$ & 0.2152 \\
\hline 71 & $(74.61,15.48)$ & $(74.1809,15.3804)$ & 0.4405 \\
\hline 72 & $(74.59,12.55)$ & $(74.0933,12.6250)$ & 0.5024 \\
\hline 73 & $(83.11,15.65)$ & $(82.5463,15.9939)$ & 0.6603 \\
\hline 74 & $(91.73,16.16)$ & $(87.8861,18.7707)$ & 4.6467 \\
\hline 75 & $(4.921,4.963)$ & $(5.0292,5.0758)$ & 0.1563 \\
\hline 76 & $(40.77,3.638)$ & $(40.3915,3.1550)$ & 0.6136 \\
\hline 77 & $(40.95,0.034)$ & $(41.4568,0.60965)$ & 0.7669 \\
\hline 78 & $(80.51,6.722)$ & $(83.2856,3.8003)$ & 4.0300 \\
\hline 79 & $(85.69,4.339)$ & $(85.1608,5.6823)$ & 1.4438 \\
\hline 80 & $(82.24,2.515)$ & $(80.5633,3.4959)$ & 1.9425 \\
\hline
\end{tabular}

between transmitter and receiver according to some deterministic law, which can be referred to as path loss model [16]. It is noted that the path loss model plays an important role in improving the quality and reliability of ranging accuracy [17]. Hence, it is needed to search an accurate path loss model to describe the relationship between RSSI and distance. In present, the traditional path loss models have been adopted to estimate the transmitter-receiver (T-R) distance $[18,19]$. Due to the impact of reflection, diffraction, and scattering, the values of received signal are affected by multipath interference [19]. The multipath interference can cause signal fluctuations with respect to the free space propagation model. Therefore, it is necessary to investigate the path loss model in actual propagation environment.

With the increasing of population, the demand for food is growing. The emergence of precision agriculture (PA) makes 
it possible to improve the yield and quality of crop. The data acquisition is the bottleneck problem in PA application, and WSN is identified as one of the best solution for data acquisition. For the research of farmland monitoring based on WSN, a particular focus has been put on wheat field since wheat is one of the major crops in the world. To better describe the monitored information from wheat field, it is necessary to know the precise geographical location of sensor nodes. In view of the characteristics of RSSI-based range measurements and particular situation of large-scale farmland, to improve the accuracy of location estimation, some RSSI-based experiments are conducted. In this study, we investigate the impact of signal propagation model on location estimation. First, we propose a new path loss model on the basis of the influence of multipath interference on signal propagation characteristics. Then, we study the accuracy and adaptability of the proposed path loss model in terms of distance estimation. Finally, we focus on the location estimation based on the proposed path loss model. To the author's knowledge, this is the first study of RSSI-based location estimation in wheat field. The findings in this report will have great significance in improving the location accuracy in large-scale farmland.

The rest of the paper can be organized as follows: Section 2 gives a basic overview of RSSI-based localization. Section 3 introduces the ranging methods and the theoretical models of RF signal propagation. The corresponding ranging experiments and location result analysis are described in Section 4. Finally, the conclusions are summarized in Section 5.

\section{Related Work}

There are some works focused on studying the RSSI-based location issues from different perspectives. For example, Kumar et al. adopted RSSI-based location technique to estimate the internode distances which further used for estimating the nodes' location [20]. They concluded that the distance-estimated error for RSSI-based location scheme in WSN is roughly identical under ideal deployment conditions. Awad et al. proposed a distance-based location technique in WSN based on RSSI measurements [21] and found that the main effect on the distance measurements is the power transmission. Alippi and Vanini proposed an RSSI-based centralized location technique for outdoor environments and found that this approach is the easiest implemented way in RSSI-based multihop location systems [22]. Subaashini et al. studied the relationship between ZigBee sensor node's RSSI values and the surrounding environment specifications for variant types of obstacles placed between transmitter and receiver [23]. They found that some RSSI value in online phase has not been fingerprinted in the training phase, and hence, the location cannot be determined. Adewumi et al. computed the internode distance of a WSN relying on RSSI-based model and discovered that the distance-estimated error in indoor environment is greater than that in outdoor environment [24]. Palazon et al. utilized an RSSI-based location scheme to study the location accuracy and how it can be affected when deploying different number of anchor nodes [25]. It is found that the location accuracy can be improved as the density of anchor nodes increases. Rasool et al. examined the performance of RSSI-based location using linear least squares algorithm [26] and found that the path loss exponent and the location coordinates performs considerably better in terms of root mean square error. Whitehouse et al. performed an experimental study of the RSSI measurements and the RSSI-based ranging in outdoor scenarios [27]. They concluded that the RSSI-based ranging can provide reasonable location performance in outdoor environments but sensitive to the environmental conditions and the experimental setup. $\mathrm{Xu}$ et al. [28] proposed an RSSI-based distance measurement model using log-normal shadowing model with dynamic variance propagation model and found that the proposed propagation model owns strong self-adaptability to various environments. Livinsa and Jayashri [29] proposed an RSSI-based location algorithm for distance estimation and location estimation under outdoor environment and indoor environment. They discovered that the performance of distance estimation in outdoor environment is better than that in indoor environment. Hamdoun et al. [30] proposed the RSSI-based location algorithm by using multiple antennas at the transmitter side, the receiver side or at the both sides to calculate the location error in indoor environment. They found that the location performance for multiple antennas at the both sides is better than that for antennas at either the transmitter or the receiver side. Chuku et al. [31] proposed an RSSIbased location scheme to mitigate the effects of shadowing caused by obstacles that are scattered in the field of operation. They found that the proposed scheme can effectively eliminate the RSSI signals that are affected by obstructions. $\mathrm{Xu}$ et al. [32] proposed an RSSI-based differential correlation algorithm to correct the distance measurement results and found that the proposed algorithm can improve the absolute accuracy as well as the relative accuracy of the location. Daiya et al. [33] performed the RSSI measurements between two sensor nodes at a varying distance for indoor and outdoor environment. They found that the estimation errors for approximate location of the sensor node are $5-10 \%$.

\section{RSSI-Based Location Method}

3.1. The Traditional RSSI-Based Ranging Model. As is known, the estimation of internode distance is the premise for node location. Herein, the accuracy of distance estimation depends heavily on the accuracy of the signal propagation model. The traditional signal propagation models include free space model (FSM), two-ray ground model, and lognormal shadowing model (LNSM). Specifically, FSM is an ideal model, and the received power decays as a function of transmitter-receiver (T-R) distance. The FSM can be expressed as follows [34]:

$$
\mathrm{PL}=20 \log (d)+20 \log (f)-27.55
$$

where PL, $d$, and $f$ are the path loss of signal energy, the signal transmission distance, and the wireless signal frequency, 


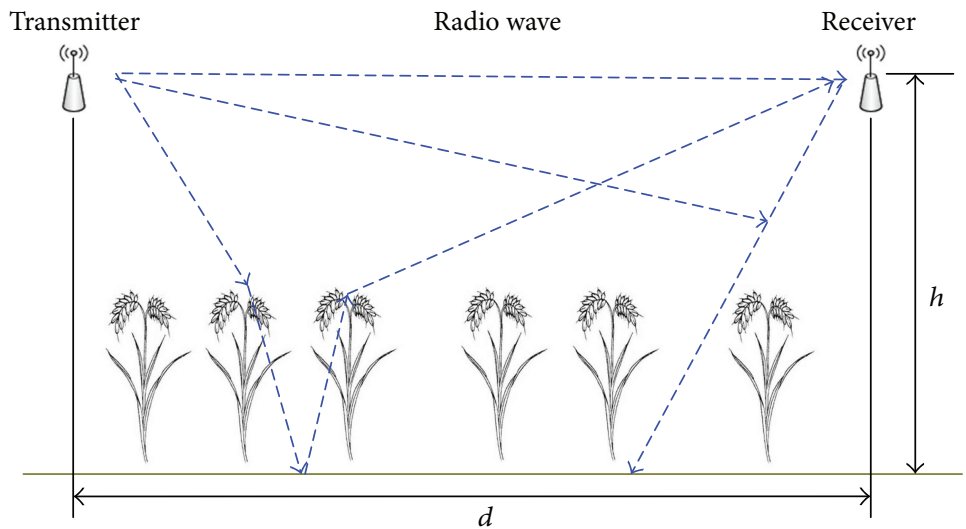

FIgURE 1: The reflection, diffraction, and scattering for radio propagation in wheat field environment.

respectively. The two-ray ground model can be represented as follows [35]:

$$
\mathrm{PL}=40 \log (d)-20 \log \left(h_{\mathrm{t}}\right)-20 \log \left(h_{\mathrm{r}}\right)
$$

where $h_{\mathrm{t}}$ and $h_{\mathrm{r}}$ are the antenna heights of transmitter and receiver, respectively. The LNSM can be represented as follows [36]:

$$
\operatorname{PL}(d)=\operatorname{PL}\left(d_{0}\right)+10 n \log \left(\frac{d}{d_{0}}\right)+X_{\sigma},
$$

where $d_{0}$ is the near-earth reference distance which depended on the experimental value, $n$ is the path loss index in a specific environment, and $X_{\sigma}$ is a zero-mean Gaussian random variable.

The mature wheat plant grows more lush and flouring, and the plant height can reach to a height of $1.2 \mathrm{~m}$. In this circumstance, the wireless signal is affected by the scattering, refection, and diffraction, which can cause multipath propagation. Due to the wheat plant usually block the path between the transmitter and receiver, the radio propagation environment in wheat field is complex. As shown in Figure 1, the radio waves arrive at the receiver from different directions, and there are slightly difference in propagation delays. It can be seen that the multipath occurs due to the reflection from ground along with reflection, scattering, and diffraction from wheat plant. The received signal at any point in space may consist of a number of waves owning randomly distributed amplitudes, phases, and angles of arrival. These multipath components are combined vectorially at the receiver, thus resulting in the distortion or fading of the received signal strength, i.e., multipath fading. That is, the multipath fading can result in the distortion of RSSI measurements, further reduce the accuracy of distance estimation. The existing empirical vegetation models include Weissberger model, ITU-R model, and COST-235 model. Concretely speaking, the Weissberger model can be represented as follows [37]:

$$
\mathrm{PL}_{\mathrm{W}}= \begin{cases}1.33 \times f^{0.284} d^{0.588}, & 14 m<d \leq 400 m, \\ 0.45 \times f^{0.284} d, & 0 m \leq d<14 m,\end{cases}
$$

where $f$ and $d$ are the frequency in $\mathrm{GHz}$ and the depth of the foliage in meter, respectively. The Weissberger model is applicable for the frequency ranging from $230 \mathrm{MHz}$ to $35 \mathrm{GHz}$. The ITU-R model can be defined as follows [38]:

$$
\mathrm{PL}_{\mathrm{ITU}-\mathrm{R}}=0.2 f^{0.3} d^{0.6},
$$

where $f$ and $d$ are the frequency in $\mathrm{MHz}$ and the depth of the foliage in meter, respectively. The ITU-R model is applicable in the situation when the frequency is ranging from $0.2 \mathrm{GHz}$ to $95 \mathrm{GHz}$. Moreover, the COST-235 model can be expressed as follows [39]:

$$
\mathrm{PL}_{\mathrm{COST}-235}=15.6 * f^{-0.009} * d^{0.26},
$$

where $f$ denotes the frequency in $\mathrm{MHz}$, and $d$ represents the depth of foliage in meter.

3.2. The Proposed Ranging Model. Based on our previous study in [40], we select the optimal fitting parametric exponential decay model (OFPEDM) as the signal propagation model in wheat field. The general form of OFPEDM can be represented as follows:

$$
\operatorname{PL}(d)=X f^{Y} d^{Z}
$$

where $f$ is the frequency of RF signal, and $d$ is the T-R distance. It is worth mentioning that $X, Y$, and $Z$ are parameters which can be determined by the actual propagation environment. Further, the estimated distance can be represented as follows:

$$
\begin{aligned}
d & =\left(\frac{\operatorname{PL}(d)}{X * f^{Y}}\right)^{1 / Z}=\left(\frac{|\overline{\operatorname{RSSI}}|}{X * f^{Y}}\right)^{1 / Z}, \\
\overline{\operatorname{RSSI}} & =\frac{1}{N} \sum_{i=1}^{N} \operatorname{RSSI}_{i},
\end{aligned}
$$

where $\mathrm{RSSI}_{i}$ represents single measurement value.

3.3. Node Location Method. To estimate the location of targeted node, this targeted node must be capable to detect at least three anchor nodes' location information. Each anchor node owns the ability to store position coordinates 
itself and the RSSI value receiving from the targeted node. The estimated distance between the targeted node and anchor node can be calculated by the proposed OFPEDM. Based on the estimated distance, we further adopt trilateration technique to estimate the position of targeted node. As presented in Figure 2, the coordinates of three anchor nodes are labeled as $\left(x_{1}, y_{1}\right),\left(x_{2}, y_{2}\right)$, and $\left(x_{3}, y_{3}\right)$, respectively. The actual distance between targeted node and three anchor nodes is denoted as $d_{1}, d_{2}$, and $d_{3}$, respectively. The circle can be given by:

$$
\left(x-x_{i}\right)^{2}+\left(y-y_{i}\right)^{2}=d_{i}^{2},
$$

where $x_{i}$ and $y_{i}$ denote the coordinates of anchor nodes. $x$ and $y$ are the coordinates of targeted nodes. Moreover, $d_{i}$ is the actual distance between targeted node and anchor node $i$. Further, the intersection of three circles can be calculated as follows:

$$
\begin{aligned}
& \left(x-x_{1}\right)^{2}+\left(y-y_{1}\right)^{2}=d_{1}^{2}, \\
& \left(x-x_{2}\right)^{2}+\left(y-y_{2}\right)^{2}=d_{2}^{2}, \\
& \left(x-x_{3}\right)^{2}+\left(y-y_{3}\right)^{2}=d_{3}^{2} .
\end{aligned}
$$

Equation (10) can be changed into the linear expression as follows:

$$
A X=B
$$

where

$$
\begin{aligned}
& A=\left[\begin{array}{ll}
-2\left(x_{1}-x_{3}\right) & -2\left(y_{1}-y_{3}\right) \\
-2\left(x_{2}-x_{3}\right) & -2\left(y_{2}-y_{3}\right)
\end{array}\right], \\
& X=\left[\begin{array}{l}
x \\
y
\end{array}\right], \\
& B=\left[\begin{array}{l}
d_{1}^{2}-d_{3}^{2}-x_{1}^{2}+x_{3}^{2}-y_{1}^{2}+y_{3}^{2} \\
d_{2}^{2}-d_{3}^{2}-x_{2}^{2}+x_{3}^{2}-y_{2}^{2}+y_{3}^{2}
\end{array}\right] .
\end{aligned}
$$

Further, the least squares solution can be expressed as follows:

$$
X=\left(A^{T} A\right)^{-1} A^{T} B .
$$

The ranging error can be defined as the difference between the estimated distance and the actual distance, which can be represented as follows:

$$
e_{i}=r_{i}-d_{i}
$$

where $r_{i}$ is the estimated distance between targeted node and anchor node $i$.

The location error of targeted node can be defined as follows:

$$
d_{e}=\sqrt{\left(x-x_{e}\right)^{2}+\left(y-y_{e}\right)^{2}}
$$

where $x$ and $y$ denote the actual coordinates of the targeted node. Moreover, $x_{e}$ and $y_{e}$ represent the estimated coordinates of the targeted node.

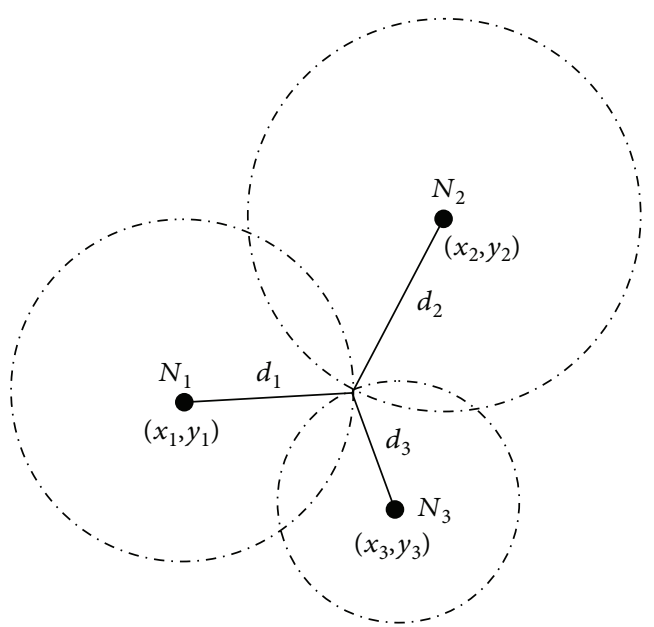

FIGURE 2: Lateration in two-dimensional coordination system with three anchor nodes.

\section{Ranging Experiments and Location Analysis}

\subsection{Ranging Experiments}

4.1.1. Experimental Setup. The ranging experiment is conducted in the wheat field in Xiaotangshan National Precision Agriculture Research Demonstration Base. The measurements adopt CC2530 wireless sensor node in Webee Company to support the ZigBee protocol at frequency of $2.4 \mathrm{GHz}$. The measurement apparatus in the experiments includes the transmitter and receiver part. During the process of measurement, the transmitter configuration is arranged in a fixed location. The measurements are taken every $2 \mathrm{~m}$ for $\mathrm{T}-\mathrm{R}$ distance ranging from $1 \mathrm{~m}$ to $100 \mathrm{~m}$. Moreover, the antenna heights of transmitter and receiver are fixed at $1 \mathrm{~m}$. The receiver supports a maximum bitrate of $250 \mathrm{kbit} / \mathrm{s}$, with $-110 \mathrm{dBm}$ of sensitivity. The RSSI measurement process is as follows: the transmitter sends a packet to the receiver and the receiver incorporates the RSSI of the received packet. Then, the receiver can forward the RSSI value to the base station which is connected with the laptop through USB cable. It is worth mentioning that the RSSI in every measuring point is recorded 100 times.

4.1.2. Distance Estimation Results Analysis. In order to gain insight into the performance of the proposed OFPEDM, we investigate the accuracy of distance estimation and adaptability to environment variations. The ranging measurement in a wheat field is depicted in Figure 3. In this circumstance, the base station is located at the edge of the field. The transmitter is placed at the source spots and moved forward in steps of $2 \mathrm{~m}$. After sampling process, the corresponding T-R distance is calculated by FSM, LNSM, and the proposed OFPEDM. Herein, $X, Y$, and $Z$ in the proposed OFPEDM are 0.051, 0.832 , and 0.253 , respectively [40]. The distance estimation results by three path loss models are illustrated in Figure 4. It can be seen that the proposed OFPEDM performs better than the FSM in terms of distance estimation accuracy for T-R distance larger than $5 \mathrm{~m}$. For FSM, as T-R distance 


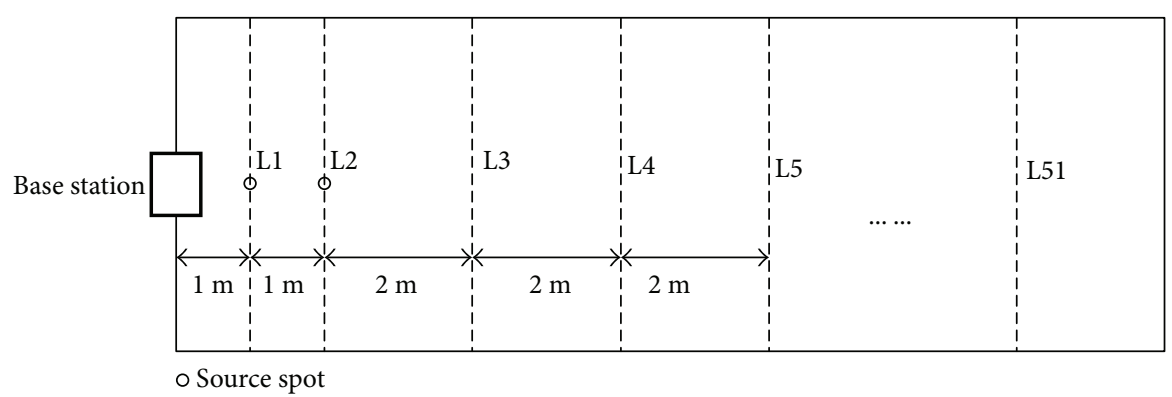

Figure 3: The ranging measurement in the wheat field. L1, L2, L3, L4, L5, ., and L51 represent the location of node 1, the location of node 2, the location of node 3 , the location of node 4 , the location of node $5, \ldots$, and the location of node 51 , respectively.

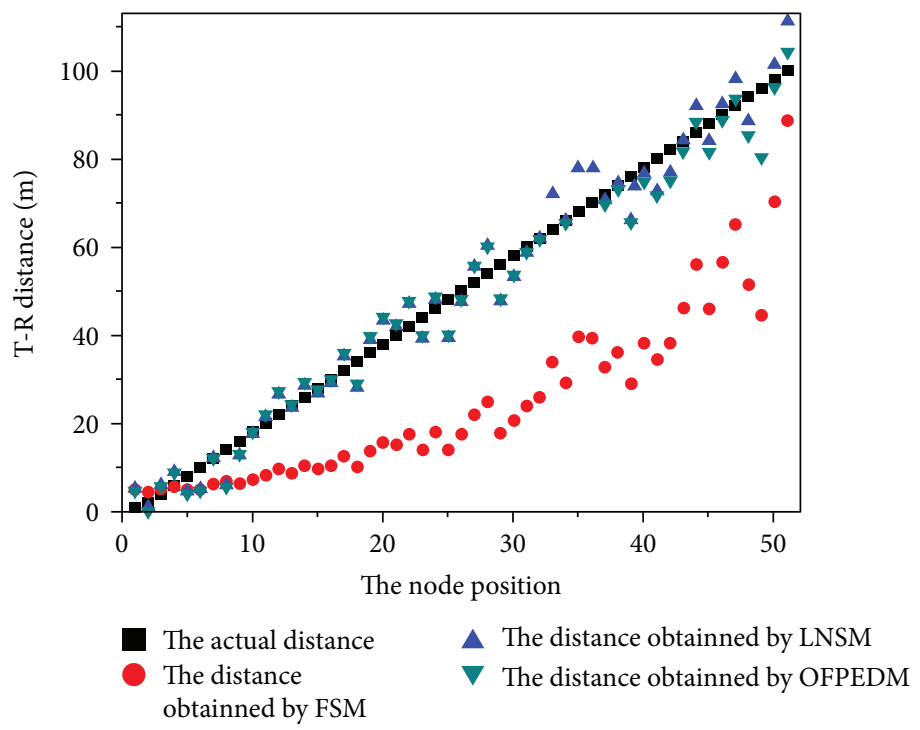

FIGURE 4: The distance estimation by FSM, LNSM, and the proposed OFPEDM.

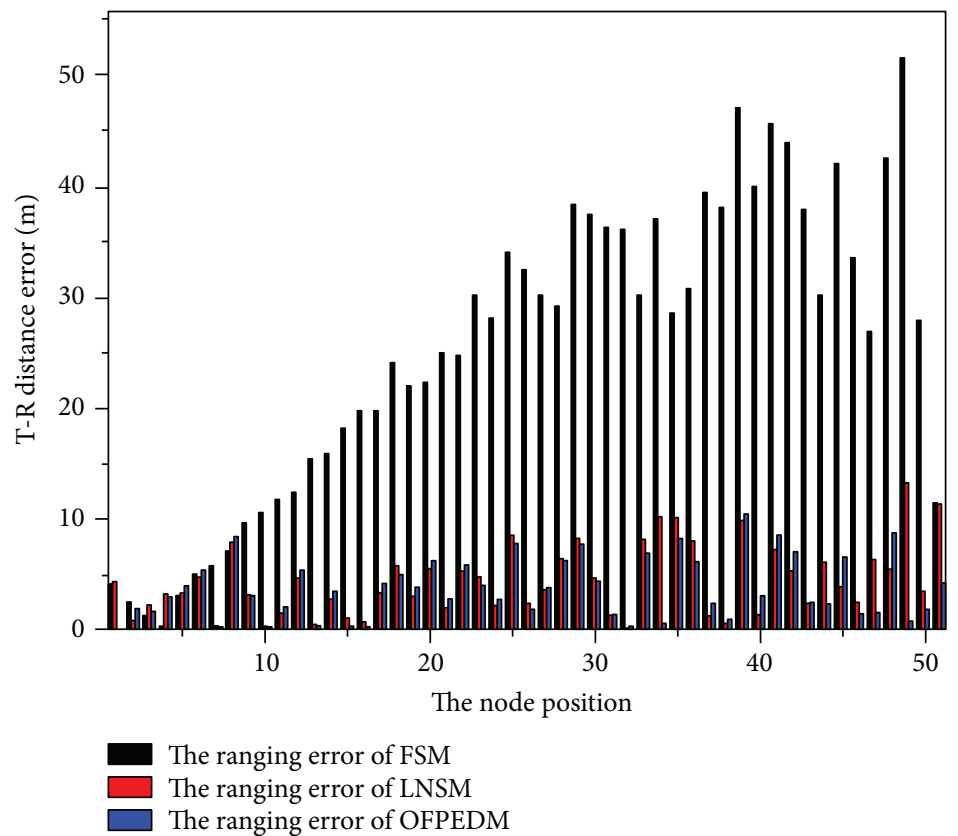

FIGURE 5: The distance estimation errors of FSM, LNSM, and the proposed OFPEDM. 


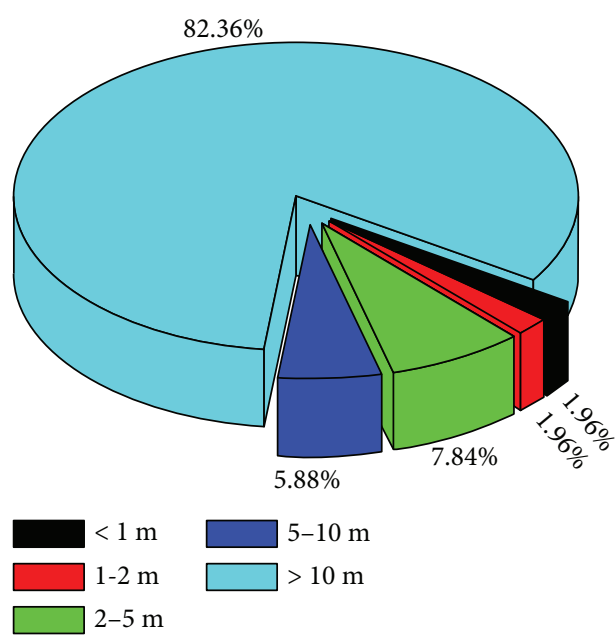

(a)

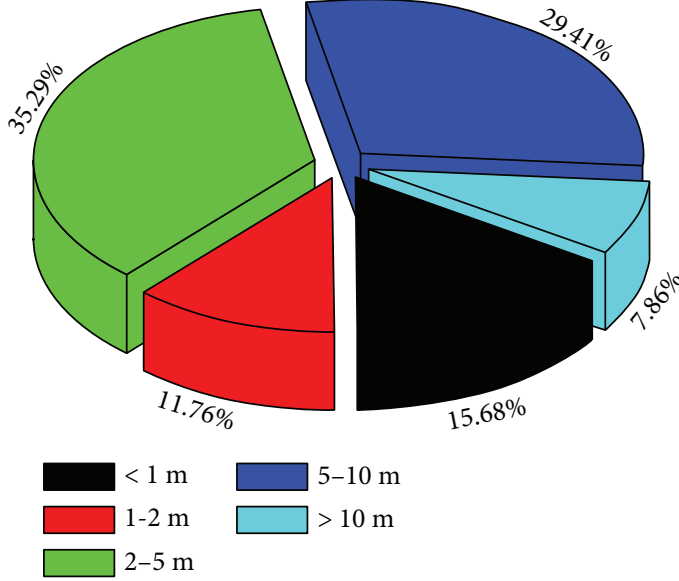

(b)

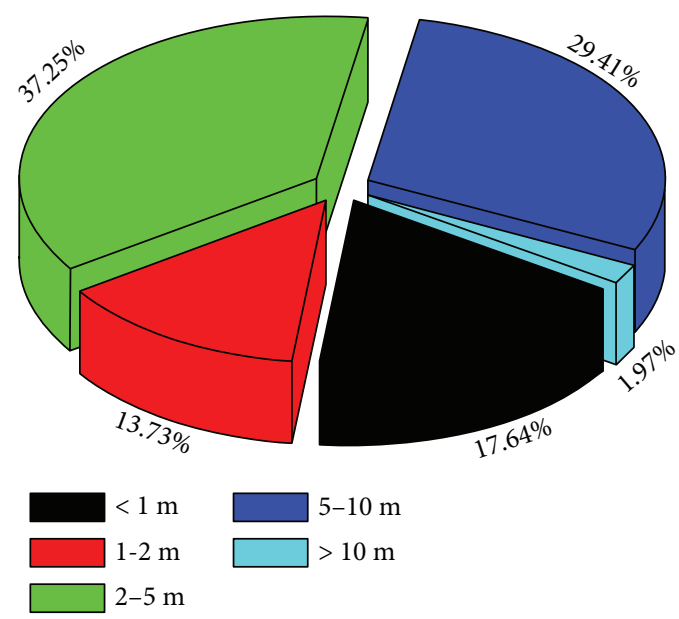

(c)

FIGURE 6: The statistical results of distance estimation error of three path loss models: (a) FSM; (b) LNSM; (c) the proposed OFPEDM.

increases, the accuracy of distance estimation fluctuates obviously and gradually gets worse since multipath fading. Compared with FSM, the proposed OFPEDM can significantly mitigate the multipath fading effect and reduce the negative effect on the accuracy of distance estimation. Moreover, the proposed OFPEDM performs better than LNSM in terms of distance estimation, which can be attributed to the fact that the proposed OFPEDM can better describe the signal propagation in wheat field.

To further evaluate the performance of three path loss models, we calculate the distance estimation errors of FSM, LNSM, and the proposed OFPEDM, as presented in Figure 5. It can be seen that the distance estimation error of FSM is always the largest, which is consistent with the distance estimation accuracy. While the distance estimation errors of LNSM and the proposed OFPEDM are relatively small. To make the distance estimation error for three path loss models more clear, we perform the statistical analysis, and the results are illustrated in Figure 6. For FSM, LNSM, and the proposed OFPEDM, the distance estimation errors smaller than $1 \mathrm{~m}$ account for $1.96 \%, 15.68 \%$, and $17.64 \%$, respectively. The distance estimation errors in the range of
0-2 $\mathrm{m}$ for FSM, LNSM, and the proposed OFPEDM are $3.92 \%, 27.44 \%$, and $31.37 \%$, respectively. The distance estimation errors smaller than $5 \mathrm{~m}$ for FSM, LNSM, and the proposed OFPEDM are $11.76 \%, 62.73 \%$, and $68.62 \%$, respectively. Moreover, the distance estimation errors smaller than $10 \mathrm{~m}$ for FSM, LNSM, and the proposed OFPEDM are $17.64 \%, 92 \%$, and $98 \%$, respectively. In brief, the distance estimation error of the proposed OFPEDM is the smallest during the ranging measurement.

To evaluate the adaptability of three path loss models to environment variations, we calculate cumulative distribution function $(\mathrm{CDF})$ of ranging errors, as presented in Figure 7. For FSM and LNSM, the probability of ranging errors smaller than $10 \mathrm{~m}$ is 0.18 and 0.92 , respectively. While for the proposed OFPEDM, the probability of ranging errors smaller than $10 \mathrm{~m}$ has been up to 0.98 . That is, the proposed OFPEDM performs better than FSM and LNSM in terms of adaptability to environment variations.

4.2. Location Estimation Results Analysis. To evaluate the performance of the RSSI-based location algorithm, the simulation experiments are achieved by MATLAB. The location 


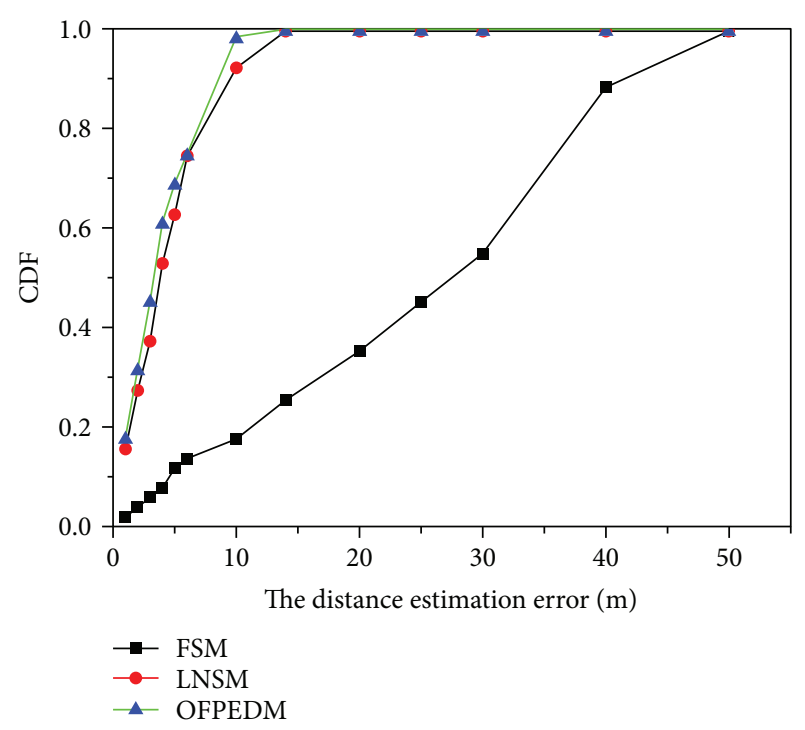

FIgURE 7: The CDF of ranging errors for FSM, LNSM, and the proposed OFPEDM.

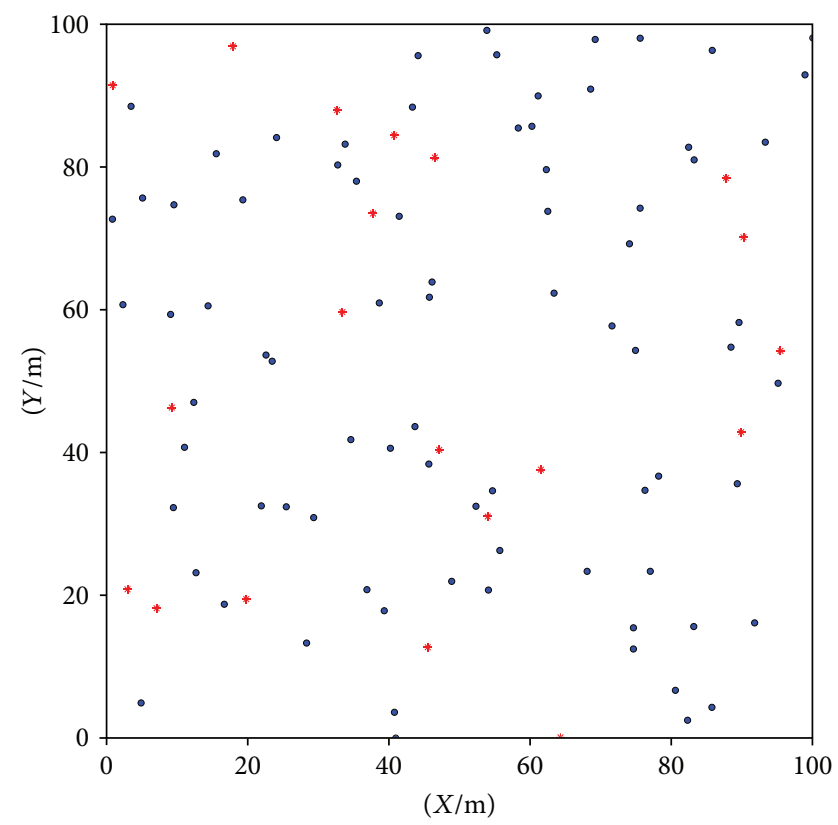

FIgure 8: The deployment of sensor nodes in the wheat field. The red "*" indicate the anchor nodes, and the blue "." represent the unknown nodes.

experiments are conducted at $100 \mathrm{~m} \times 100 \mathrm{~m}$ region area. The schematic of node deployment is presented in Figure 8 . The number of anchor nodes is 20 , and the number of targeted nodes is 80 . The actual coordinates of anchor nodes are $(0.933,91.50),(17.92,96.89),(32.68,88.03),(40.75,84.45)$, (46.50, 81.40), (87.72, 78.49), (37.75, 73.50), (90.20, 70.21), $(33.43,59.66),(95.41,54.28),(9.299,46.35),(89.84,42.92)$, $(47.11,40.4),(61.53,37.66),(54.01,31.11),(3.039,20.85)$, (19.82, 19.51), (7.123, 18.2), (45.50, 12.73), and (64.27, 0.142 ), respectively. Moreover, the communication radius for each node is set to $30 \mathrm{~m}$.

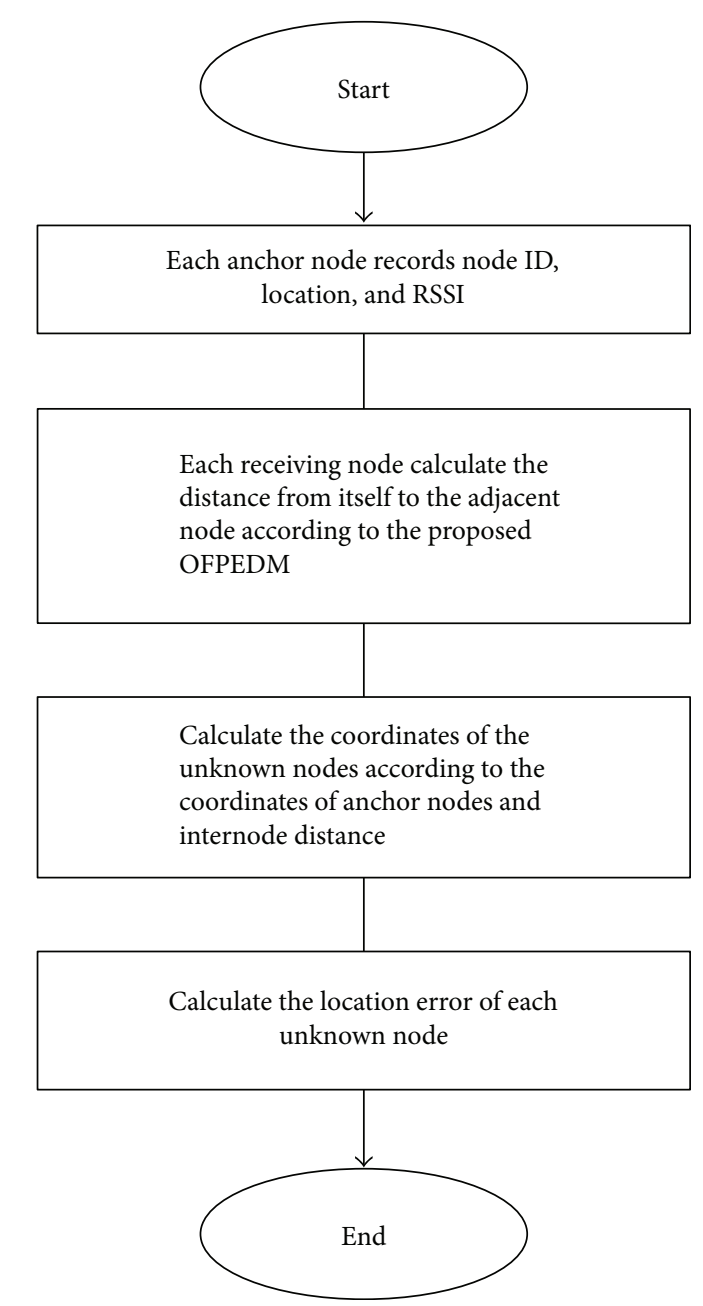

FIgURE 9: The flow chart of RSSI-based location algorithm.

The flow chart of RSSI-based location algorithm is presented in Figure 9. Through simulating in MATLAB, the location results of unknown nodes are presented in Figure 10. As listed in Table 1, the location estimation errors for all the unknown nodes range from $0.0004 \mathrm{~m}$ to $5.1739 \mathrm{~m}$. Moreover, the location estimation error decreases as the targeted node moving towards the center of the field, which can be attributed to the fact that the density of anchor nodes in the center of the field is larger than that in the edge of the field.

\section{Conclusions}

Through the analysis of ranging experiments performed at the wheat field, we find that the proposed OFPEDM can achieve a higher estimation accuracy and adaptability to environment variations in comparison to the FSM and LNSM. Hence, we adopt OFPEDM to describe the relationship between RSSI value and the internode distance. To evaluate the performance of the RSSI-based location algorithm, the simulation experiments are achieved by MATLAB. For all the unknown nodes, the location errors range from $0.0004 \mathrm{~m}$ to $5.1739 \mathrm{~m}$. The location error of RSSI-based method is influenced not only by the location algorithm, 


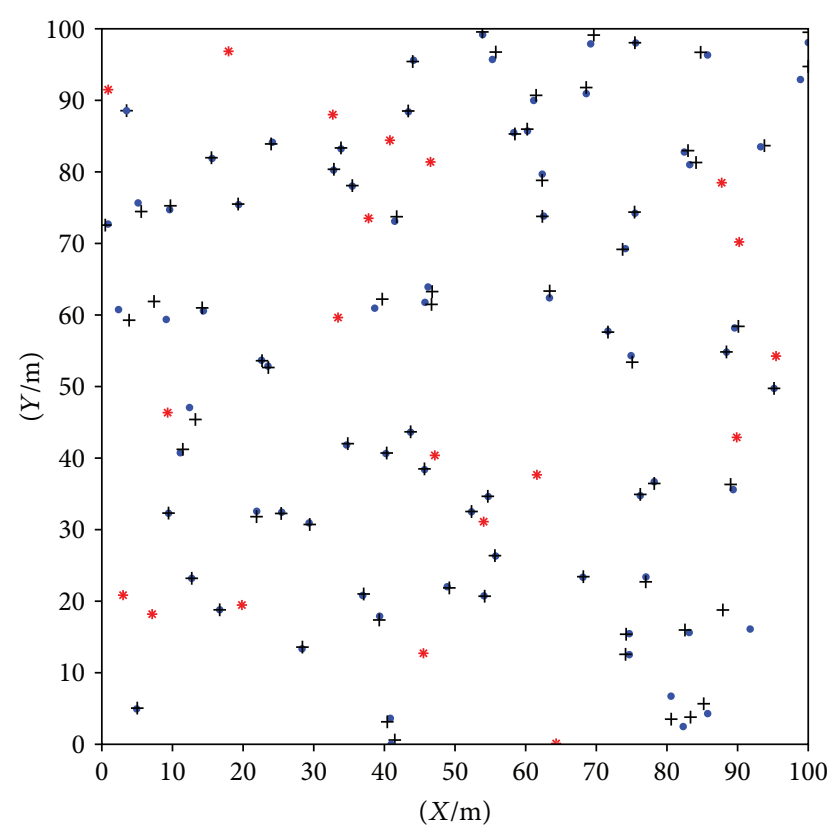

Figure 10: The location results of the unknown nodes. The red " *" indicate the position of anchor nodes, the blue "." represent the actual position of unknown nodes, and the black "+" denote the estimated position of unknown nodes.

but also by the ranging accuracy. The location error in this RSSI-based location algorithm is acceptable in the wide areas such as wheat field. The findings in this research may provide reference for location estimation in large-scale farmland.

\section{Conflicts of Interest}

The authors declare that they have no conflicts of interest.

\section{Acknowledgments}

This work is supported by the Beijing Natural Science Foundation (4172024) and Natural Science Foundation of China (61571051).

\section{References}

[1] X. Wang, S. Wang, and J. J. Ma, “An improved particle filter for target tracking in sensor systems,” Sensors, vol. 7, no. 12, pp. 144-156, 2007.

[2] G. Mao, B. Fidan, and B. D. O. Anderson, "Wireless sensor network localization techniques," Computer Networks, vol. 51, no. 10, pp. 2529-2553, 2007.

[3] T. Yang and $\mathrm{X}$. Wu, "Accurate location estimation of sensor node using received signal strength measurements," $A E U$ International Journal of Electronics and Communications, vol. 69, no. 4, pp. 765-770, 2015.

[4] Z. Yang and Y. Liu, "Quality of trilateration: confidence-based iterative localization," IEEE Transactions on Parallel and Distributed Systems, vol. 21, no. 5, pp. 631-640, 2010.

[5] J. Zhao, W. Xi, Y. He et al., "Localization of wireless sensor networks in the wild: pursuit of ranging quality," IEEE Transactions on Networking, vol. 21, no. 1, pp. 311-323, 2013.
[6] I. Guvenc and C. C. Chong, "A survey on TOA based wireless localization and NLOS mitigation techniques," IEEE Communications Surveys \& Tutorials, vol. 11, no. 3, pp. 107-124, 2009.

[7] J. Shen, A. F. Molisch, and J. Salmi, "Accurate passive location estimation using TOA measurements," IEEE Transactions on Wireless Communications, vol. 11, no. 6, pp. 2182-2192, 2012.

[8] F. K. Chan and H. C. So, "Accurate distributed range-based positioning algorithm for wireless sensor networks," IEEE Transactions on Signal Processing, vol. 57, no. 10, pp. 41004105, 2009.

[9] L. Cong and W. Zhuang, "Hybrid TDOA/AOA mobile user location for wideband CDMA cellular systems," IEEE Transactions on Wireless Communications, vol. 1, no. 3, pp. 439-447, 2002.

[10] H. J. Shao, X. P. Zhang, and Z. Wang, "Efficient closed-form algorithms for AOA based self-localization of sensor nodes using auxiliary variables," IEEE Transactions on Signal Processing, vol. 62, no. 10, pp. 2580-2594, 2014.

[11] A. Coluccia, "Reduced-bias ML-based estimators with low complexity for self-calibrating RSS ranging," IEEE Transactions on Wireless Communications, vol. 12, no. 3, pp. 1220 1230, 2013.

[12] S. Salari, S. Shahbazpanahi, and K. Ozdemir, "Mobility-aided wireless sensor network localization via semidefinite programming," IEEE Transactions on Wireless Communications, vol. 12, no. 12, pp. 5966-5978, 2013.

[13] Q. Luo, X. Yan, J. Li et al., "DEDF: lightweight WSN distance estimation using RSSI data distribution-based fingerprinting," Neural Computing and Applications, vol. 27, no. 6, pp. 15671575, 2016.

[14] B. C. Seet, Q. Zhang, C. H. Foh, and A. C. Fong, "Hybrid RF mapping and Kalman filtered spring relaxation for sensor network localization," IEEE Sensors Journal, vol. 12, no. 5, pp. 1427-1435, 2012.

[15] J. A. Jiang, X. Y. Zheng, Y. F. Chen et al., "A distributed RSS-based localization using a dynamic circle expanding mechanism," IEEE Sensors Journal, vol. 13, no. 10, pp. 37543766, 2013.

[16] A. Zanella, "Best practice in RSS measurements and ranging," IEEE Communications Surveys \& Tutorials, vol. 18, no. 4, pp. 2662-2686, 2016.

[17] R. B. Zhang, J. G. Guo, F. H. Chu, and Y. C. Zhang, "Environmental-adaptive indoor radio path loss model for wireless sensor networks localization," AEU-international Journal of Electronics and Communications, vol. 65, no. 12, pp. 10231031, 2011.

[18] W. Y. Chung, B. G. Lee, and C. S. Yang, "3D virtual viewer on mobile device for wireless sensor network-based RSSI indoor tracking system," Sensors and Actuators B: Chemical, vol. 140, no. 1, pp. 35-42, 2009.

[19] Q. Luo, X. Yan, J. Li, and Y. Peng, "DDEUDSC: a dynamic distance estimation using uncertain data stream clustering in mobile wireless sensor networks," Measurement, vol. 55, pp. 423-433, 2014.

[20] P. Kumar, L. Reddy, and S. Varma, "Distance measurement and error estimation scheme for RSSI based localization in wireless sensor networks," in 2009 Fifth IEEE Conference on Wireless communication and sensor networks (WCSN), pp. 1-4, IEEE, Allahabad, India, 2009.

[21] A. Awad, T. Frunzke, and F. Dressler, "Adaptive distance estimation and localization in WSN using RSSI measures," in 
10th Euromicro Conference on Digital system design architectures, methods and tools (DSD 2007), pp. 471-488, IEEE, Lubeck, Germany, 2007.

[22] C. Alippi and G. Vanini, "A RSSI-based and calibrated centralized localization technique for wireless sensor networks," in Fourth Annual IEEE International Conference on Pervasive Computing and Communications Workshops (PerCom Workshops 2006), pp. 5-305, IEEE, Pisa, Italy, 2006.

[23] K. Subaashini, G. Dhivya, and R. Pitchiah, "ZigBee RF signal strength for indoor location sensing-experiments and results," in 2013 15th international Conference on Advanced communication technology (ICACT), pp. 50-57, IEEE, PyeongChang, South Korea, 2013.

[24] O. G. Adewumi, K. Djouani, and A. M. R. S. S. I. Kurien, "RSSI based indoor and outdoor distance estimation for localization in WSN," in 2013 IEEE international conference on Industrial technology (ICIT), pp. 1534-1539, IEEE, Cape Town, South Africa, 2013.

[25] J. A. Palazon, J. Gozalvez, M. Sepulcre, and G. Prieto, "Experimental RSSI-based localization system using wireless sensor networks," in 2012 IEEE 17th Conference on Emerging Technologies \& Factory Automation (ETFA 2012), pp. 1-4, Krakow, Poland, 2012, IEEE.

[26] I. Rasool, N. Salman, and A. H. Kemp, "RSSI-based positioning in unknown path-loss model for WSN," in Sensor Signal Processing for Defence, pp. 1-5, IET, London, UK, 2013.

[27] K. Whitehouse, C. Karlof, and D. Culler, "A practical evaluation of radio signal strength for ranging-based localization," ACM SIGMOBILE Mobile Computing and Communications Review, vol. 11, no. 1, pp. 41-52, 2007.

[28] J. Xu, W. Liu, F. Lang, Y. Zhang, and C. Wang, "Distance measurement model based on RSSI in WSN," Wireless Sensor Network, vol. 2, no. 8, pp. 606-611, 2010.

[29] Z. M. Livinsa and S. Jayashri, "Performance analysis of diverse environment based on RSSI localization algorithms in wsns," in 2013 IEEE Conference on Information \& Communication Technologies (ICT), pp. 572-576, IEEE, Thuckalay, Tamil Nadu, India, 2013.

[30] S. Hamdoun, A. Rachedi, and A. Benslimane, "Comparative analysis of RSSI-based indoor localization when using multiple antennas in wireless sensor networks," in 2013 International Conference on Selected topics in mobile and wireless networking (MoWNeT), pp. 146-151, IEEE, Montreal, QC, Canada, 2013.

[31] N. Chuku, A. Pal, and A. Nasipuri, “An RSSI based localization scheme for wireless sensor networks to mitigate shadowing effects," in 2013 Proceedings of IEEE, Southeastcon, pp. 1-6, IEEE, Jacksonville, FL, USA, 2013.

[32] W. Xu, Z. Zhang, and G. Wan, "An RSSI-based differential correlation algorithm for wireless node localization," The Open Automation and Control Systems Journal, vol. 5, no. 1, pp. 7379, 2013.

[33] V. Daiya, J. Ebenezer, S. A. V. S. Murty, and B. Raj, "Experimental analysis of RSSI for distance and position estimation," in 2011 International Conference on Recent trends in information technology (ICRTIT), pp. 1093-1098, Chennai, Tamil Nadu, India, 2011, IEEE.

[34] G. Eason, B. Noble, and I. N. Sneddon, "On certain integrals of Lipschitz-Hankel type involving products of Bessel functions," Philosophical Transactions of the Royal Society A: Mathematical, Physical and Engineering Sciences, vol. 247, no. 935, pp. 529-551, 1955.
[35] K. K. Sharma and S. D. Joshi, "Signal separation using linear canonical and fractional Fourier transforms," Optics Communications, vol. 265, no. 2, pp. 454-460, 2006.

[36] A. Ghasemi and E. S. Sousa, "Asymptotic performance of collaborative spectrum sensing under correlated lognormal shadowing," IEEE Communications Letters, vol. 11, no. 1, 2007.

[37] M. Weissberger, R. Meidenbauer, H. Riggins, and S. Marcus, Radio Wave Propagation: a Handbook of Practical Techniques for Computing Basic Transmission Loss and Field Strength, 1982, DTIC Document.

[38] Y. S. Meng and Y. H. Lee, "Investigations of foliage effect on modern wireless communication systems: a review," Progress In Electromagnetics Research, vol. 105, pp. 313-332, 2010.

[39] Committee C M, COST 235 Radiowave propagation effects on nextgeneration fixed-services terrestrial telecommunications systems, Publication and European, EU, 1996.

[40] H. R. Wu, L. H. Zhang, and Y. S. Miao, "The propagation characteristics of radio frequency signals for wireless sensor networks in large-scale farmland," Wireless Personal Communications, vol. 95, no. 4, pp. 3653-3670, 2017. 


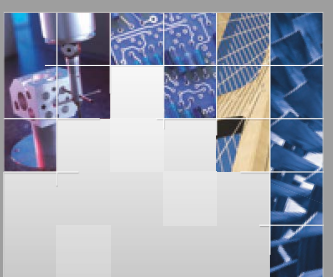

\section{Enfincering}
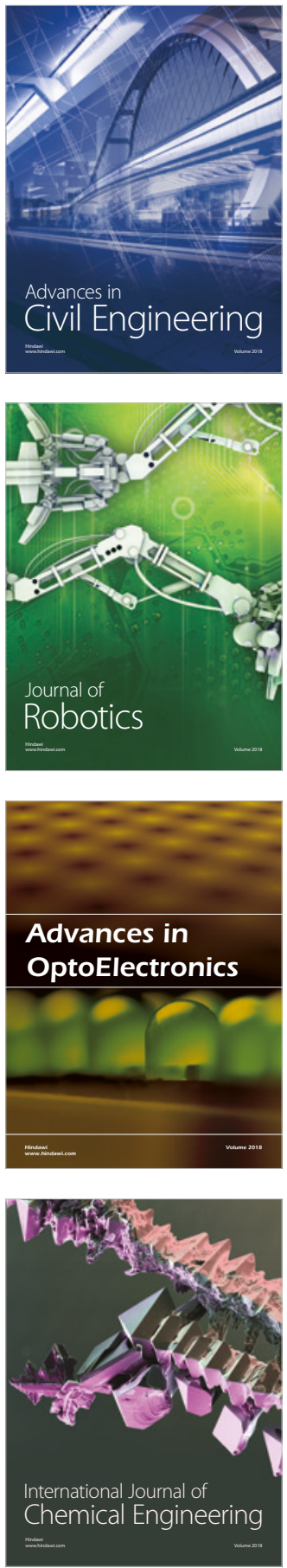

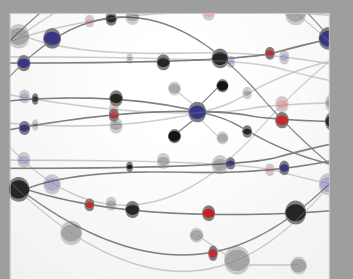

\section{Rotating \\ Machinery}

The Scientific World Journal

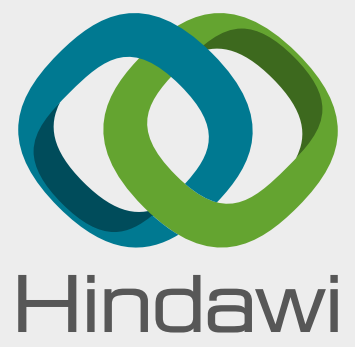

Submit your manuscripts at

www.hindawi.com
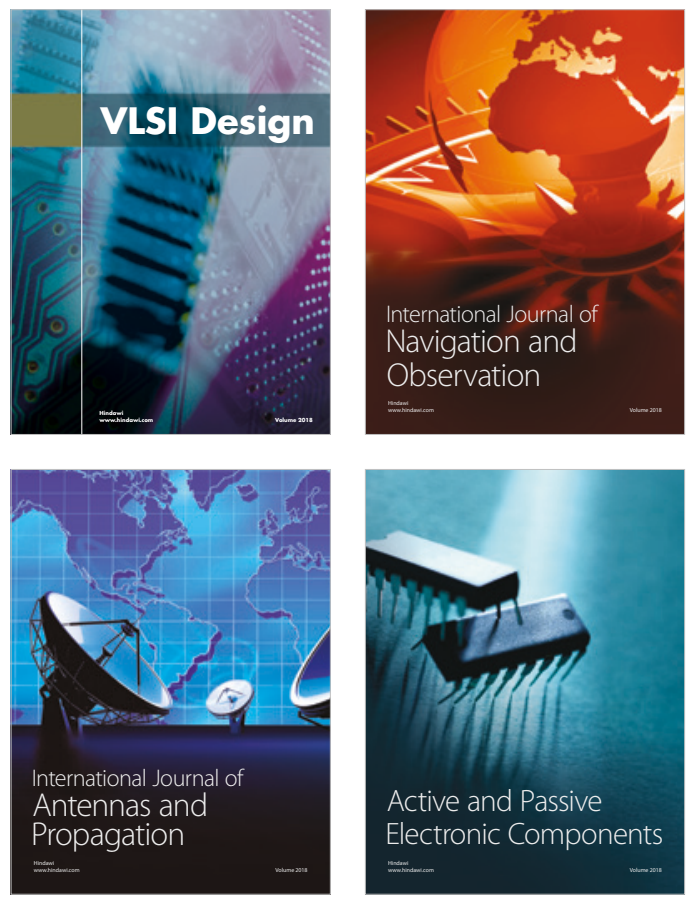
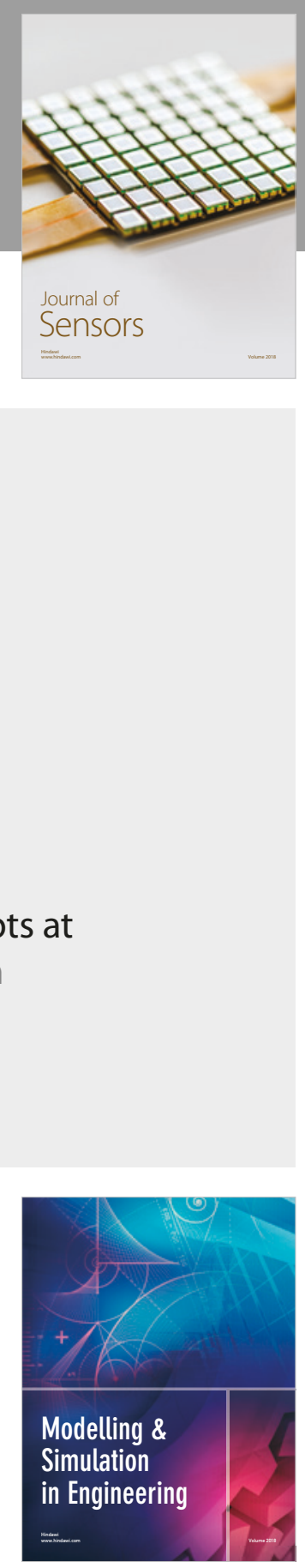

\section{Advances \\ Multimedia}
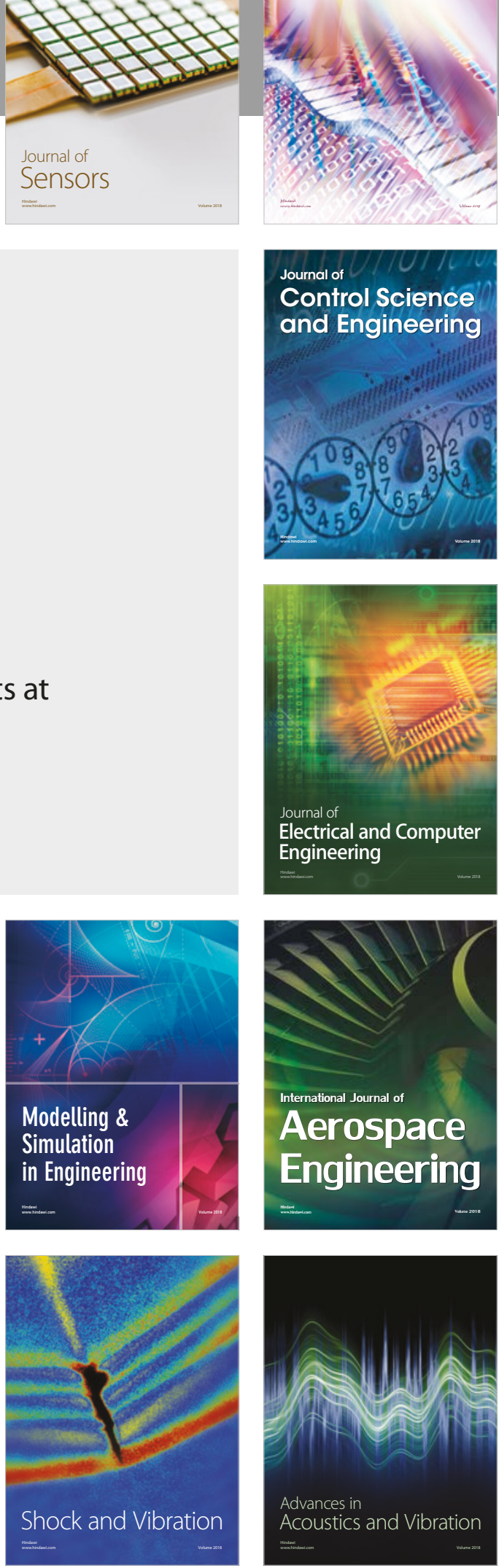